\title{
Topological Determinants of Synchronizability of Oscillators on Large Complex Networks
}

\author{
Weihan $\mathrm{Li}^{1}$ and Changsong Zhou*,1,2
}

\author{
${ }^{I}$ Department of Physics, Hong Kong Baptist University, Hong Kong \\ ${ }^{2}$ Centre for Nonlinear Studies and The Beijing-Hong Kong-Singapore Joint Centre for Nonlinear and Complex Systems \\ (Hong Kong), Hong Kong Baptist University, Kowloon Tong, Hong Kong
}

\begin{abstract}
Synchronized oscillations play an important role in many biological systems. In recent years, much work has been done on oscillating biomolecular systems, both experimentally and theoretically. A better insight into oscillation mechanisms, coupling strategies and related biological processes is gained by quantitative analysis. Here we summarized some of recent work on oscillation and synchronization in biological systems and reviewed the basic concepts of synchronization of coupled oscillators and dynamics on complex networks.
\end{abstract}

Keywords: Systems biology, dynamical modeling, complex networks, oscillations.

\section{INTRODUCTION: OSCILLATION AND SYNCHR- ONIZATION IN BIOMOLECULAR SYSTEMS}

Timing is crucial for biological systems. Oscillation carries the information of time. Such property makes oscillation important in biology. In order to exhibit biological autonomous oscillations, two elements, namely negative feedback and time delay, are usually, but not always, essential $[1,2]$. A negative feedback prevents the process from either going too fast or too slow while sufficient time delay allows for an overshoot. Together, they make oscillation possible. If either one is missing, the system loses the oscillating property. The classic population model illustrates this idea very well. If the species reproduces very fast, like bacteria, the carrying capacity of the environment has an instant effect on the population. The population will eventually come to a stable fixed point. However, if the species reproduces with a relatively longer period, like sheep-blowfly [3], a time delay $\tau$ should be introduced into this dynamical equation of the system because the carrying capacity of the environment would affect the population after time $\tau$. The experimental and simulation results show that the system obtains oscillatory behavior after the time delay is added.

Many biological oscillations are subject to negative feedback with time delay. One of the best examples comes from glycolytic oscillation [4-6]. The inhibition of ATP on the enzyme phosphofructokinase (PFK), which is an enzyme in reaction $V_{1}$ in Fig. (1), is the negative feedback (Fig. 1). When the flow of the pathway is large, more ATP would be generated and in turn inhibits PFK to slow down the reaction. When the flux is small, however, ATP inhibition would decrease and the reaction accelerates. The

\footnotetext{
*Address correspondence to this author at the Centre for Nonlinear Studies and The Beijing-Hong Kong-Singapore Joint Centre for Nonlinear and Complex Systems (Hong Kong), Hong Kong Baptist University, Kowloon Tong, Hong Kong; Tel: (852) 3411 5089; Fax: (852) 3411 5813;

E-mail: cszhou@hkbu.edu.hk
}

intermediate steps of glycolysis provide the other significant factor --- time delay. At high ATP concentration, the negative feedback slows down the reaction, but ATP concentration would not instantaneously drop because it takes time for the information of the deceleration of PFK catalysed reaction, which appears at the beginning in glycolysis, to pass through the pathway. Downstream intermediate reactions need to slow down one by one before ATP drops. Other biological systems also share the same mechanism. Respiratory oscillation is believed to be caused mainly by the inhibition of sulfate uptake, which appears at the beginning of the sulfate assimilation, by amino acid cystein, which is an end product of the pathway. This inhibition acts as the negative feedback while the intermediate steps provides the time delay $[5,7,8]$. p53Mdm2 oscillation is another example, in which gene regulation and gene expression delay are the two basic factors mentioned above. Intracellular calcium concentration oscillation in T-cells is also suggested to be related to the delayed interaction between calcium stores and CRAC channel $\left(\mathrm{Ca}^{2+}\right.$-released-activated $\mathrm{Ca}^{2+}$ channels $)$ [9]. Translational and transcriptional feedback are found to enhance the circadian oscillations in cyanobacteria and plants [10-14]. More generally, the dynamics and modelling of a class of negative feedback loops, which is common in biological circuits, is studied recently and revealed some common features of this type of oscillation [1,2]. Besides a negative feedback with time delay, additional feedbacks will result in a shape or period difference. The simplest case is genetic relaxation oscillator, a combined oscillatory effect of a fast positive feedback and a slow negative feedback. Instead of a smooth harmonic periodic curve, genetic relaxation oscillator leads to a pulse-like shape --- with a sudden rise and a slow drop. In addition, more than one negative feedback loops are sometimes employed to establish oscillation, like the Notch-Hes7-Lfng loops, found in somite segmentation, where three negative feedback loops together give rise to the oscillation in Hes7 and Lfng expression levels $[15,16]$. 


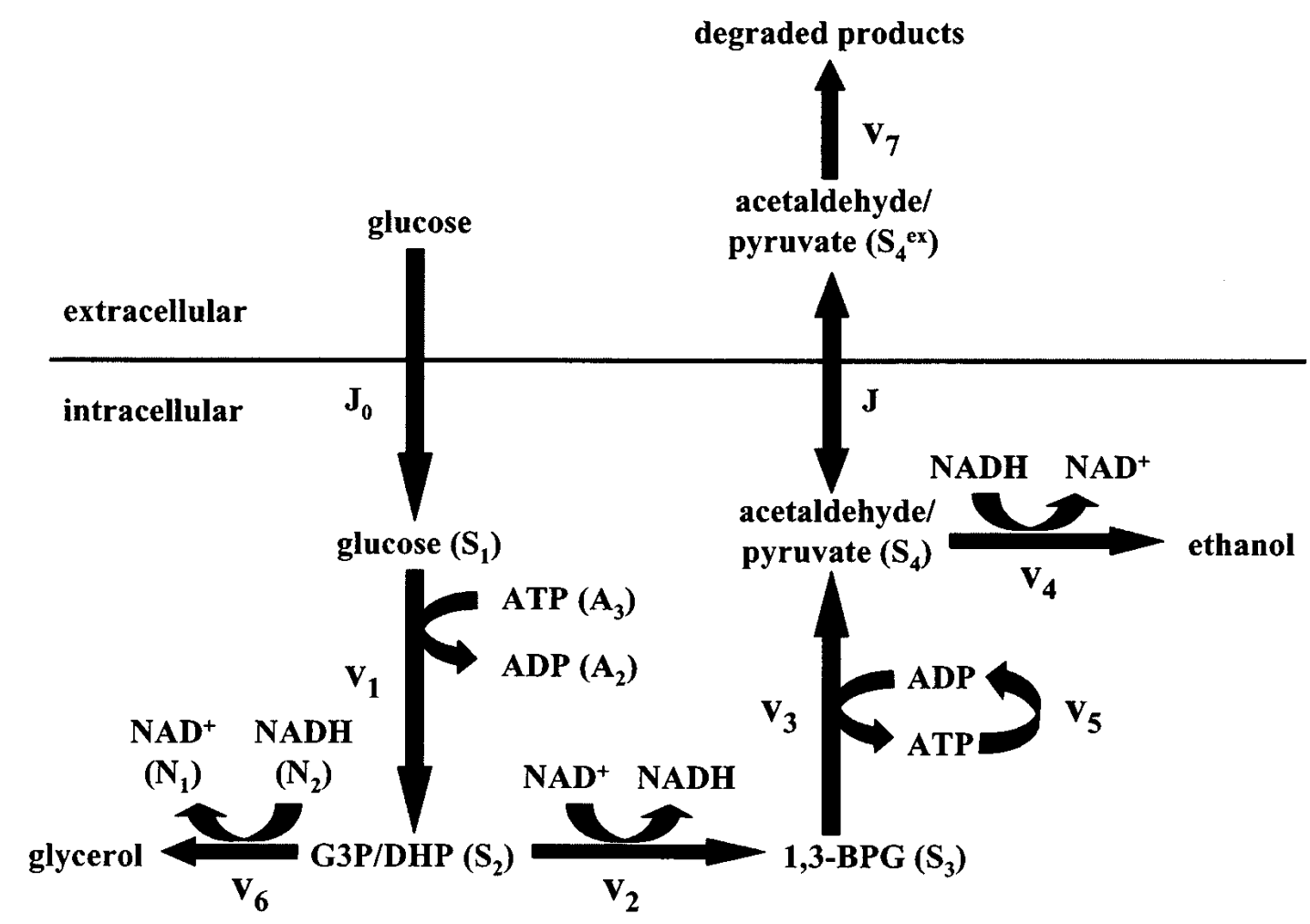

Fig. (1). Simplified metabolic network for modeling yeast glycolytic oscillations [5, 6].

Now we know the strategies biological systems employed to establish oscillations. However, many biological ensembles, usually consisting of hundreds, thousands or even more oscillators, carry out processes that require accurate timing. To carry information of time in a population of cells or a multicellular organism, oscillation of individual oscillators must be synchronized. Oscillations without synchronization would lead to an overall effect in a mess as each one may have its phase and intrinsic natural frequency. For example, consider hurrying people on a busy street in rush hours, each has a distinct pace regardless of the others --- some may be running because of being later, while others are in normal pace. Thus, the pace information of the overall population can be hardly told. Nevertheless, such disordered outcome is avoided in a marching troop where paces are all highly synchronized.

So, what are the strategies that biological systems employed to achieve synchronization? Generally speaking, two mechanisms are employed: information exchange between different oscillators, or entrainment to a common external stimulus.

To illustrate such strategies, imagine two people, say James and John, walking side by side and initially having different natural frequency, i.e. natural pace in this case. If they simply walk on their own, regardless of the other one's pace, they will end up in their intrinsic paces and can be treated separately as two independent subsystems. However, if they walk in such a way that one tends to follow the other one's pace, i.e. James speeds up if he sees John is faster, decelerates if John is slower and vice verse, a synchronous effect is likely to happen between these two subsystems. Another approach is that the two are walking in response to a whistle, i.e. an external stimulus we previously mentioned, paces will be unified to the whistle frequency.

Both mechanisms are often employed by biological systems. The synchronization of glycolytic oscillation within a cell population is believed to be related to the cell-cell communication with acetaldehyde (Aca) [17-19]. Respiratory oscillation may be, suggested by some experiments, closely related to the hydrogen sulfide mediation within the population $[5,7,8]$. Many other genetic oscillators, including some synthetic gene networks, are coupled by intercellular exchange of certain small compound. Information exchange between different oscillators, however, does not have to be intercellular. In vitro experiments indicate that KaiC monomers exchange between different hexamers, which belongs to intracellular signal exchange, couples the phosphorylation status of the ensemble within a cell. Still, intercellular information exchange is more common in biological systems. Similarly, the second synchronizing mechanism is also important and frequently encountered in biological systems. The most remarkable example is the circadian clock in fungal and plant $[12,20]$. Light, as a common external stimulus that changes periodically day and night, enters the pathway by a photoreceptor and couples the corresponding oscillator.

So far, we have discussed the strategies that biological systems choose to establish oscillations and to synchronize a population of oscillators. If we treat each oscillator as a node and interaction between oscillators as an edge, a network is obtained. A crucial question is: how does the system behavior depend on the network structure? To explore it, we begin with the phase oscillator model and the simplest 
structure --- all-to-all connected network. After that, we will study the impact of complex network structure.

\section{PHASE OSCILLATOR MODEL}

Research on synchronization started early in the 17th century when the famous physicist Christiaan Huygens observed a "kind of sympathy", later known as synchronization, in two pendulum clocks "so constructed from two hooks embedded in the same wooden beam". He later reported his observation that "the motions of each pendulum in opposite swings were so much in agreement that they never receded the least bit from each other and the sound of each was always heard simultaneously. Further, if this agreement was disturbed by some interference, it reestablished itself in a short time. For a long time I was amazed at this unexpected result, but after a careful examination finally found that the cause of this is due to the motion of the beam, even though this is hardly perceptible" [21].

Despite the long history of the study of synchronization, a major breakthrough was made only decades ago by Winfree [22, 23] when he represented biological oscillators as simple phase oscillators, regardless of amplitudes as they are hardly influenced under weak coupling. With the phase oscillator model, each system is fully determined by a timedependent phase variable $\theta$ whose evolution is influenced by the phases of other interacting oscillators.

The examples of two people walking in pace can be mathematically described by the following equations:

$\dot{\theta}_{1}=\omega_{1}+F_{1}\left(\theta_{1}, \theta_{2}\right)$,

$\dot{\theta}_{2}=\omega_{2}+F_{2}\left(\theta_{1}, \theta_{2}\right)$.

Here, $\theta_{1}, \theta_{2}$ are the phases, or paces in this case, of two oscillators while $\omega_{1}, \omega_{2}$ are their natural frequencies. $F_{1}$ and $F_{2}$, acting as the coupling term, are periodic functions of $\theta_{1}$ and $\theta_{2}$ and have the property that if $\theta_{1}>\theta_{2}$, then $F_{1}<0$, $F_{2}>0$, and if $\theta_{1}<\theta_{2}$, then $F_{1}>0, F_{2}<0$. Such properties allow the phase difference, $\left|\theta_{1}-\theta_{2}\right|$, to get smaller and smaller so that the paces are eventually locked with $\left|\theta_{1}-\theta_{2}\right|$ $=$ const, i.e. the two oscillators are synchronized.

Synchronization by external signals is quantitatively described by

$\dot{\theta}_{1}=\omega_{1}+G_{1}\left(\theta, \theta_{1}\right)$

$\dot{\theta}_{2}=\omega_{2}+G_{2}\left(\theta, \theta_{2}\right)$,

where $\theta$ is the phase of external stimulus. $G_{1}, G_{2}$ are positive if $\theta_{1}<\theta, \theta_{2}<\theta$, and are negative if they are the other way round.

The above situations correspond to the two approaches to obtain synchronization. They can be further generalized into $N$ oscillators, where $N$ is usually very large in biological systems. The internal interaction coupling follows $\dot{\theta}_{j}=\omega_{j}+\sum_{l} F_{j, l}\left(\theta_{j}, \theta_{l}\right)$

The external stimulation coupling is governed by

$\dot{\theta}_{j}=\omega_{j}+G_{j}\left(\theta, \theta_{j}\right)$.

Here, $F_{j, l}$ and $G_{j}$ have similar property as we mentioned in the two-oscillator case.

The periodic coupling functions $F$ and $G$ can be expanded into Fourier series. If we take the simplest form $F_{j, l}\left(\theta_{j}, \theta_{l}\right)=\sin \left(\theta_{l}-\theta_{j}\right)$ and $G_{j}\left(\theta, \theta_{j}\right)=\sin \left(\theta-\theta_{j}\right)$, we get the well known Kuramoto model [24]. A great deal of understanding of synchronization of oscillators has been obtained analytically by the Kuramoto model [24, 25].

\section{SYNCHRONIZATION IN ALL-TO-ALL CON- NECTED NETWORKS}

In biology, there are many cases where interaction exists within every pair of oscillators. The best studied example is glycolytic oscillation. As previously introduced, each cell can establish glycolytic oscillation. Thus, every cell can be treated as an oscillator. Oscillators are coupled by a small metabolite Aca [17-19]. As a small metabolite that can penetrate the cell membrane freely, Aca secreted by each cell forms a pool in the extracellular environment (Fig. 1). The Aca pool oscillates as a result of the oscillation in each cell. Aca in the extracellular environment can also flow into each cell to influence their phases and amplitudes [17]. Each individual oscillator is interacting with another through the Aca pool. Therefore, the whole population forms an all-to-all connected network. Moreover, according to [30], Aca diffusion rate should be much faster than the time scale of intracellular dynamics, corresponding to a strong coupling among the cells. Many other biological systems, such as respiratory oscillation, also establish all-to-all connected network in a similar way. So what is the synchronizability on such all-to-all connected network?

Here, we look into this question through the Kuramoto model, which describes the synchronous behavior among a large number, $N$, of interacting phase oscillators. Each oscillator, fully determined by a phase variable $\theta_{j}$, has a natural frequency $\omega_{j}$ that obeys a distribution density $g(\omega)$. Then oscillators will run at their own natural frequencies, but with a phase-dependent feedback information from the rest that tends to couple the system. More precisely, the dynamics of the ensemble follows

$\dot{\theta}_{j}=\omega_{j}+\frac{K}{N} \sum_{l=1}^{N} \sin \left(\theta_{l}-\theta_{j}\right)$.

Here, $K$ is the coupling constant that quantifies the coupling strength within the system, determined, for example, by the diffusion rate of Aca in glycolytic oscillations. To be more illustrative, let us imagine the phase oscillators as a group of people running around a unit circular track with intrinsic angular velocity $\omega_{j}$. Then what Eq. (7) tells us is that for any two individuals $j$ and $l, j$ 
tends to slow down a little to wait for $l$ if $j$ is about half a circle ahead, and tends to speed up to catch up with $l$ if it is about half a circle behind, and vice verse for $l$. Eq. (7) follows the general form of Eq. (5).

Notice that every pair of oscillators in the ensemble interacts with each other in the same way -- with the same dynamic function and same coupling constant $K$. Thus, this all-to-all connected system (fully connected network) has symmetry and can be tackled with mean field theory [24, $25]$. To quantify the collective behavior of the population, an order parameter, $r(t)$, and an average phase, $\varphi(t)$, are introduced as

$r e^{i \varphi}=\frac{1}{N} \sum_{l=1}^{N} e^{i \theta_{l}}$.

Interpreted into our illustrative racing example, the point $r e^{i \varphi}$ gives center of mass of the group of people. If the group is nonsynchronized, runners will be almost evenly distributed on the track with various angular velocities, and the center of mass will be close to $r=0$. On the other hand, if it is synchronized, the runners will end up running hand in hand with almost the same angular velocity and phase, and the center of mass is close to the circular track. Thus, $r(t)$, ranging from 0 to 1 , is a measurement of the coherence of the system. $r=0$ and $r=1$ correspond to the limiting cases of totally incoherent and phase-locked cases respectively.

Multiply both sides of Eq.(8) by $e^{-i \theta_{j}}$, the imaginary parts of it becomes

$r \sin \left(\varphi-\theta_{j}\right)=\frac{1}{N} \sum_{l=1}^{N} \sin \left(\theta_{l}-\theta_{j}\right)$.

Substituding it back into Eq.(7) gives rise to

$\dot{\theta}_{j}=\omega_{j}+K r \sin \left(\varphi-\theta_{j}\right)$.

From Eq.(10), it is easy to see that the interactions between oscillators can be completely replaced by the influence of a mean-field through the quantities $r$ and $\varphi$. What's more, Eq.(10) shows that if the coupling strength $K$ is very small, i.e. the second term on the right-hand side is negligible, then it is reduced to

$\dot{\theta}_{j}=\omega_{j}$.

Almost all the oscillators run at their natural frequencies, and the population is not synchronized. On the other hand, if $K \gg>\frac{\omega_{j}}{r}$, then the reduced form of Eq. (10) is

$\dot{\theta}_{j}=K r \sin \left(\varphi-\theta_{j}\right)$.

$\theta_{j}=\varphi$ would be a stable fixed point which corresponds to such a system behavior that all oscillators are phaselocked at a common frequency and the order parameter $r \rightarrow 1$. In between, $r$ increases monotonically with $K$. When $K$ reaches a certain threshold, the order parameter will have a sudden increase as it is shown in Fig. (2). This is because of the positive feedback of $r$ in Eq.(10) -- when $r$ is large, the second term $K r \sin \left(\varphi-\theta_{j}\right)$ contributes more to make the system more coherent. As a result, $r$ increases and enhances the effect of $\operatorname{Kr} \sin \left(\varphi-\theta_{j}\right)$.

More detailed analysis and numerical solutions [24, 25] show that:

(i)

Oscillators that have natural frequencies $\omega_{j}<K r$ will end up with their phases locked, and they oscillate with a common frequency $\Omega$.

(ii) Oscillators that have natural frequencies $\omega_{j}>K r$ will end up running at frequencies around their natural frequencies.

In many physical or biological systems, the behavior of a single oscillator can be hardly detected through experiments. Often, only the averaged quantity of the population is measurable. The overall oscillation depends on $r$, i.e. the coherence of the system to a great extend. Only when the system is highly synchronized, can the oscillation of the population occur, see Fig. (3). Therefore, synchronization of real biological systems can be manifested in experiments by pronounced oscillations of the concentration of certain measurable component. An example is shown in Fig. (4) for the yeast glycolytic oscillations. Here oscillations of the NADH fluorescence indicate synchronized oscillations with a population. When two populations with anti-phase oscillations are mixed, synchronization of the cells is temporally lost and the collective oscillations of the mixed population vanish for a while and appear soon when synchronization is re-established in the new population. For such a well studied system, a set of equations can be written down based on the metabolic network in Fig. (1) and synchronization can be studied quantitatively [4-6]. Importantly, most of the features of synchronization observed in experiments and in simulations of the models

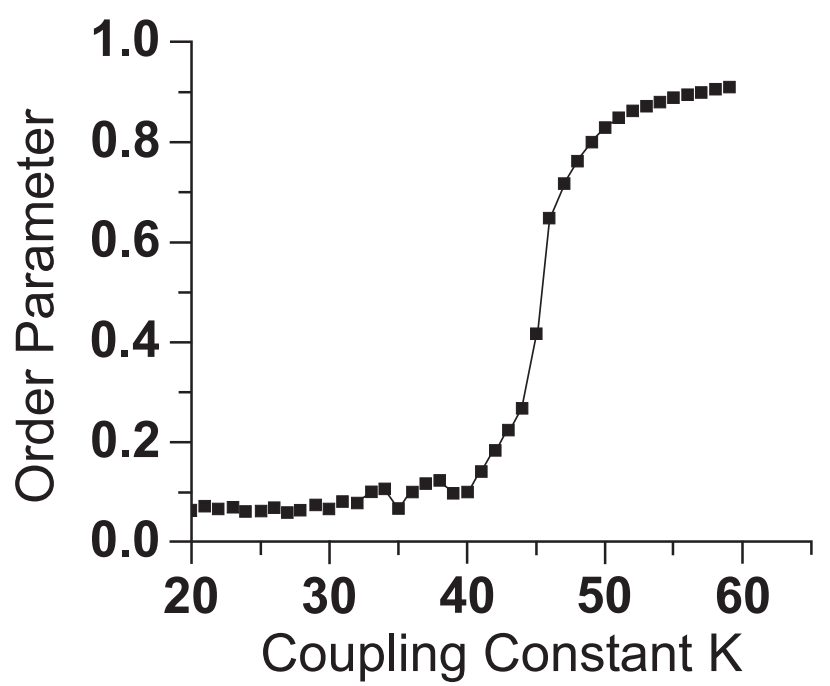

Fig. (2). The order parameter $r$ as a function of the coupling strength $K$. As the coupling constant increase, the system becomes more coherent. Here, the natural frequency distribution of this 500oscillator system follows the normal distribution with a bias of 50 and variance of 10 . 

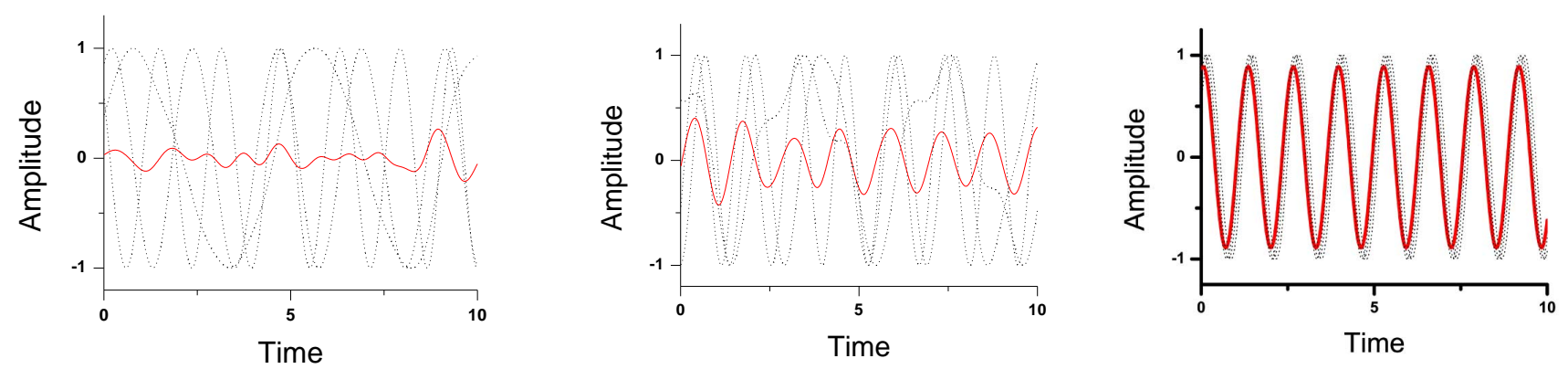

Fig. (3). Synchronization of an ensemble of phase oscillators, $N=500$, at different coupling strengths. (a) $K=35, r=0.083$, oscillators basically run at their own natural frequencies as the coupling strength is too weak. No periodic population behavior (average over individual time series) is observed. (b) $K=45, r=0.416$, some oscillators begin to synchronize, and averaged oscillation emerges. (c) $K=55$, $r=0.887$, almost all oscillators are in phase, thus the population average is basically the same as that of a single oscillator.

can be understood using the simple Kuramoto model in the all-to-all connected networks.

Now we have seen the system behavior in a simplest network structure, namely all-to-all connected network. Next, we are going to study the dependence of the synchronizability on some more complex network structures.

\section{SYNCHRONIZATION IN COMPLEX NETWORKS}

So far, we have addressed of dynamics on all-to-all connected systems. However, there are also many cases where the architecture is much more complex. Networks, which are now a common representation in the natural and social sciences, are comprised of large number of nodes and edges. They may carry different meanings in various networks. Take a few biological networks for example. In metabolic networks, usually nodes are metabolites and edges are reactions. For neural networks, nodes can be a single neuron or a set of locally well connected neurons, and edges would be the interaction between them. In gene regulatory networks, nodes are mRNAs levels and the edges represent the causal influences between them. In protein interaction networks the nodes are proteins and the edges represent their molecular interactions.

The circadian rhythm in mammals, a daily variation of $24 \mathrm{~h}$ that regulates basic physiological processes [26], which is governed by a central clock in brain called suprachiasmatic nucleus ( $\mathrm{SCN}$ ) [27], serves as a good example of synchronization in complex networks. SCN is composed of a population of one kind of neuron cells known as clock cells, which are self-sustained oscillators. The clock cells are connected in a complex manner rather than fully connected. SCN establishes oscillation even without external signals, due to synchronization among the clock cells, but such oscillation has a longer period, about $25 \mathrm{~h}$ in human, than a day [28]. In addition, SCN also employs the other strategy for synchronization, namely entrainment to an external stimulus. A subset of neurons in SCN receives light signals from the environment [29], which helps adjust the period and phase of SCN oscillation so that it is locked with local time.

Mammalian brain is a complex system where oscillations, synchronization and complex network topology are all relevant. Reliable databases are available now for large-scale systems level connectivity formed by long-range projections among cortical areas in the brains of several animals $[31,32]$. Large-scale anatomical brain networks are densely connected, with very complex and heterogeneous connectivity patterns [33-35]. The activity of the brain observed experimentally by electroencephalographs (EEG) or functional magnetic resonance imaging (fMRI), is

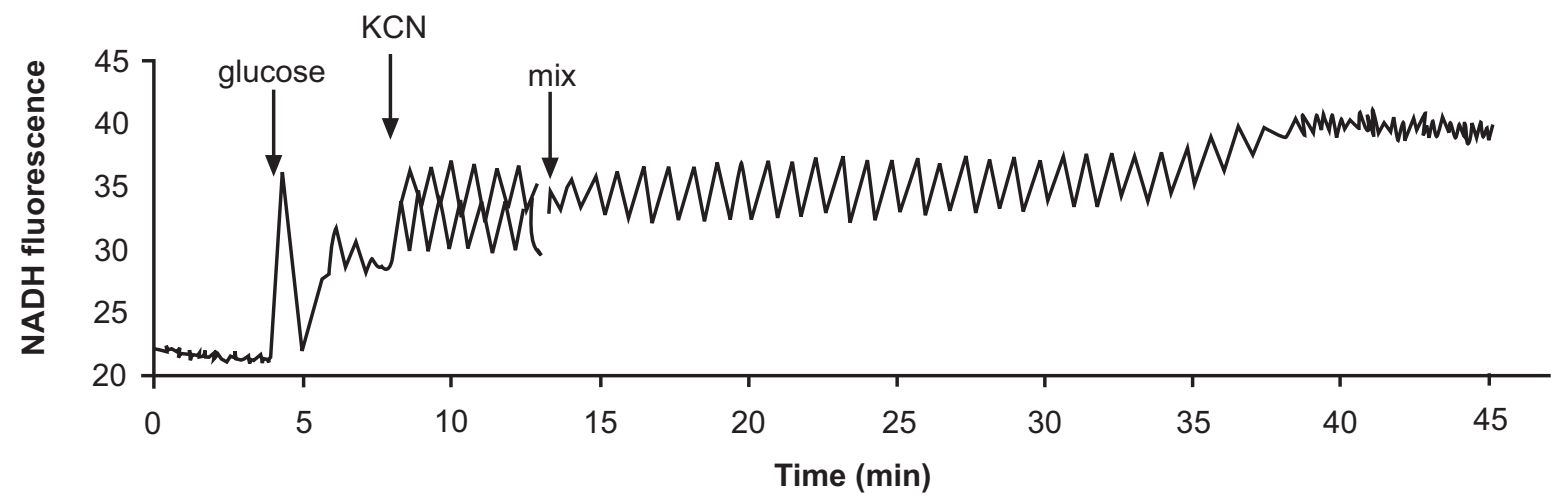

Fig. (4). Synchronization of yeast glycolytic oscillations in mixing experiment. Two suspensions of yeast cells, each with a glycolytic oscillation, were at first cultured separately. The phase difference between them was managed to set to 180 degrees. At a certain moment, mix the two suspensions. The oscillation of total nicotinamide adenine dinucleotide hydride(NADH) concentration was barely observable in the first 1-2 min, but after around 5-6min, i.e. 7-8 periods of oscillation, a synchronized oscillation reappeared, with the same period and similar amplitude as the one before mixing. From [4]. 
characterized by oscillations occurring over a broad spectrum and by synchronization over a wide range of spatial and temporal scales [36]. The interplay between the complex network architecture and complicated oscillations form complex functional networks of the brain [37-40]. Understanding large-scale organization of the brain from the viewpoint of complex networks [32, 41, 42] has become a multidisciplinary topic of great interest.

Such biological examples highlight the need for studying synchronization in networks with more complex architecture. The Kuramoto model on complex networks leads to a remarkable understanding for oscillatory ensembles. Coherence depends both on coupling strength and network topology. So, before going into detailed dynamical analysis, it is necessary to have a brief introduction on complex network topology. More systematic reviews of complex networks can be found in [43-45]. In our case, i.e. Kuramoto model on complex network, each node represents an oscillator, and edges stand for the interactions. Network topology usually influences the dynamic behavior in various ways. Two common and well discussed types of network are scale-free network [46] and small-world network [47]. Scale-free networks have the property that the nodes' degree distribution follows power law which indicates that no particular scale can be set to the network. Smallworld network arises from the famous small world experiment, which states that one can be reached from any other within a few steps or edges as in fully random networks, even though the network is highly clustered locally. Such networks usually require several long range connections between different communities in which nodes are densely connected. When phase oscillators are placed on a complex network, such as scale-free network or smallworld network, interesting coherence phenomena emerge within the ensemble.

In order to obtain a quantitative analysis, the generalized Kuramoto model is introduced, which is governed by

$\dot{\theta}_{i}=\omega_{i}+\frac{K}{N} \sum_{j} a_{i j} \sin \left(\theta_{j}-\theta_{i}\right)$,

where $a_{i j}$ are the elements of the connectivity matrix. When all $a_{i j}=1(i \neq j)$ the original Kuramoto model in all-to-all connected network is recovered.

Studies on synchronization in complex topologies were first reported for small-world and scale-free networks. These works are mainly numerical explorations of the onset of synchronization beyond which groups of nodes oscillating coherently first appear. The surprising results show that a critical point exists when the network is described by a power-law connectivity distribution with an exponent $\gamma>3$

[48]. Beyond such critical point, the system is fully synchronized.

For degree heterogeneous random networks, in which the degree distribution can be broad, but the connections among the oscillators are otherwise random, theoretical approaches argued that the critical coupling is proportional to $\langle k\rangle /\left\langle k^{2}\right\rangle$ [49]. As a result, different topologies should give rise to distinct critical points to synchronization. In particular, the path towards synchronization in Erdös-Renyi random networks and scale-free networks has been compared in [50] (see Fig. 5). In fact, the onset of synchronization first occurs for scale-free networks. As the network substrate becomes more homogeneous, the critical point shifts to larger values and in this sense the networks seem to become less synchronizable. However, the amplitude of the collective oscillation of the network is larger in Erdös-Renyi random networks. The reason is that in scale-free network, the nodes with large degrees are more strongly influenced by the mean field so that synchronization among these subsets of nodes can be achieved with weaker couplings. When the coupling becomes stronger, almost all the oscillators are synchronized in Erdös-Renyi random networks, but many nodes with small degrees in the scale-free networks are still not synchronized, leading to a smaller collective oscillation.

The topology of complex networks not only influences the transition point to coherence, but also changes the route that the oscillators undergo the transition to synchronization. In globally coupled oscillators, before the system achieves global locking of frequency and phases, oscillators with frequencies close to each other first form a synchronized cluster. However, in a complex network, such effective cluster is not solely determined by frequency, but can also be strongly influenced by the network topology. As seen in Fig. (5), the transition to complete synchronization, $r=1$, is sharper for homogeneous networks. The reason is that the formation of effective synchronization clusters is different in random and scale-free networks, as shown in Fig. (6). In scale-free network, both links and nodes are incorporated together to the largest synchronized cluster formed by hubs, while for random network, links are added to the nodes already belonging to a cluster.

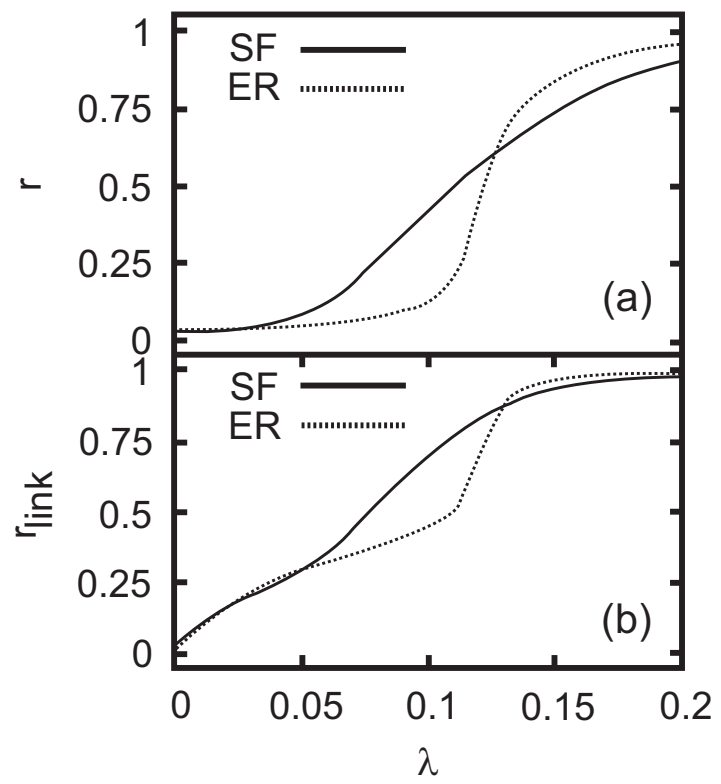

Fig. (5). Comparison of the transition to synchronization in random network (ER) and scale-free (SF) networks. Here $\lambda$ is the coupling strength. $r$ is the order parameters over the all the nodes and $r_{\text {link }}$ measures the synchronization between the nodes with network connections. From [50]. 


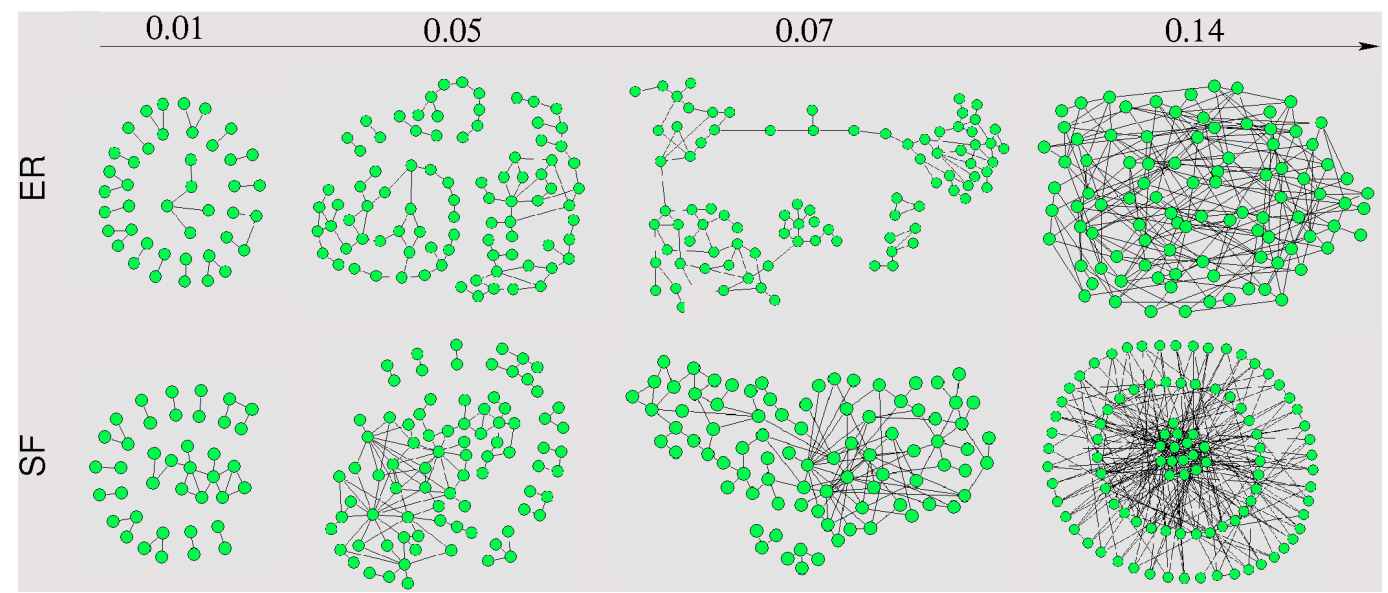

Fig. (6). Synchronized clusters for several values of $\sigma$ for the two cases of random and scale-free networks. From [50].

As we discussed before, there are two schemes for synchronization: mutual interaction and entrainment by external stimulus. Many biological processes are subjected to both schemes. An good example is circadian rhythms generated by complex network of neurons in SCN [27] where the neurons are coupled through a complex network connectivity and part of the neurons are subject to direct influence of photic input [29]. The entrainment of coupled phase oscillator networks by external pacemaker has been studied in [51, 52]. It is shown that the entrainment frequency window of a network decreases exponentially with the depth of the network. The depth is defined as the average forward distance of the elements from the pacemaker. These results show that only shallow networks can be effectively locked by the pacemaker. This analysis is of great importance to understand the function of biological systems in a changing environment.

The concept of synchronization on complex networks has been expanded significantly over the last few years [53]. It is not just restricted to the study of phase oscillators only. In fact, a more general situation, where the oscillators can display more complicated oscillations, such as chaotic oscillators, can be considered. In such cases, even two identical oscillators with different initial conditions will follow totally different trajectories in phase space and thus do not synchronize. However, coupling between the oscillators can stabilize the trajectory and the oscillators can follow the same orbits in phase space while the orbits can still be very complex and chaotic. Theoretically, the complete synchronization of a whole network of identical oscillators is suitable for theoretical analysis and has been the focus of research in the field of dynamics of large scale complex networks. Even though such theoretical setting may not be very close to real systems, the analysis provides fundamental insights on how the network topology determines dynamical patterns on networks. Therefore, we provide a brief review of this topic as well, and the readers can refer to [53] for a more comprehensive review.

The dynamics of each oscillator is governed by a set of equations, which are assumed to be identical for all oscillators.
$\dot{x}=F(x)$.

In a network, each oscillator would send out signals, generated by an output function from the state $x, H(x)$, which is again assumed to be oscillator-independent. We can take the yeast glycolytic oscillation as an example. The time evolution of metabolites (the system variables $x$ ) in Fig. (1), such as ATP/ADP and NAD/ NADH, is described by a set of kinetic equations [5, 6], which form the functions $F(x)$. The concentration of Aca which depends on the other metabolites and is responsible for the cell-cell communication, is the output function $H(x)$ of the oscillators. Of course, in such a real systems, the cells cannot be fully identical, and the interaction is assumed to be all-to-all, but not a complex network.

The dynamics of a network of $N$ oscillators takes the form

$$
\begin{aligned}
& \dot{x}_{i}=F\left(x_{i}\right)+\sigma \sum_{i=1}^{N} a_{i j} w_{i j}\left[H\left(x_{j}\right)-H\left(x_{i}\right)\right] \\
& =F\left(x_{i}\right)-\sigma \sum_{j=1}^{N} G_{i j} H\left(x_{j}\right),
\end{aligned}
$$

where $a_{i j}=0$ or 1 represents the network topological connections, and $w_{i j}$ is the weight of the link. The combination of them is shown by the coupling matrix $G=\left(G_{i j}\right)$, which is a weighted matrix and is in general asymmetrical even though the underlying topological matrix $A=\left(a_{i j}\right)$ can be symmetrical. Mathematically, the complete synchronization state is a solution of this equation where the states of all the oscillators in the network are exactly the same, i.e.

$x_{1}(t)=x_{2}(t)=\ldots=x_{N}(t)=s(t)$,

where $s(t)$, the synchronized trajectory, is the same as that of a single oscillator, i.e., it is a solution of Eq. (14). However, such a synchronization state can be stable or unstable. It is stable when the system will return back to this state if it is perturbed away from it; otherwise, it is unstable if any small perturbation will make it impossible to come 
back. Therefore, the central question in the study of synchronization in complex networks is: under what condition the complete synchronization state is stable so that it can be realized starting from some initial conditions of the system.

The analysis is based on the observation of two coupled oscillators shown in Fig. (7a), where an oscillator $x$ is driven by another oscillator $s$. In general, the synchronization state $x(t)=s(t)$ is stable and can be achieved if the coupling strength is suitable within a range of $\alpha_{1}<\alpha<\alpha_{2}$ for typical oscillators $F$. This is a typical case for nonlinear oscillators, especially chaotic oscillators, that synchronization state of two coupled oscillators only becomes stable when the coupling strength $\alpha$ is larger than the threshold $\alpha_{1}$, and usually should be smaller than another threshold $\alpha_{2}$. The thresholds $\alpha_{1}$ and $\alpha_{2}$ depends on the particular oscillators $F$, as well as the coupling function $H$, and in some cases the synchronization region can be unbounded, i.e., $\alpha_{2}=\infty$. Theoretically, using modedecomposition, we can break a complex network in Eq. (16) effectively into $N-1$ pairs of oscillators, all connected to a single oscillators $s$, as shown in Fig. (7b). The connection strength for these pairs is now $\sigma \lambda_{l}(l=2,3, \cdots, N)$, where $\lambda_{l}$ is the $l$ th eigenvalue of the network coupling function $G$ (we always have $\lambda_{1}=0$ ). To achieve the complete synchronization state of the network in Eq.(17), all the $N-1$ oscillators in Fig. (7b) should be synchronized by the common forcing $s(t)$. This means that $\alpha_{1}<\sigma \lambda_{l}<\alpha_{2}$ for all $l$. Listing all the eigenvalues of the network and ordering them, we get the condition for synchronization of a whole network as [54]:

$\alpha_{1}<\sigma \lambda_{2} \leq \sigma \lambda_{3} \leq \cdots \leq \sigma \lambda_{N}<\alpha_{2}$.

From the above equation, it is clear to see how network structure affects synchronization of oscillators. Because we hope to have a smaller coupling strength $\sigma$ to satisfy the first condition $\sigma \lambda_{2}>\alpha_{1}$, the larger the minimal non-zero eigenvalue $\lambda_{2}$ is, the easier the network could be synchronized. On the other hand, if $\lambda_{N}$ is too large, it is possible that for any $\sigma$ satisfying the first condition $\sigma \lambda_{2}>\alpha_{1}$, the second condition $\sigma \lambda_{N}<\alpha_{2}$ cannot be fulfilled and synchronization cannot be achieved at all. To find a region of $\sigma$ where the synchronization could be realized, the ratio of the eigenvalues must satisfy $R=\lambda_{N} / \lambda_{1}<\alpha_{2} / \alpha_{1}$. This analysis shows that when considering complex networks, the synchronization dynamics becomes quite complicated: the same set of oscillators ( $\alpha_{1}$ and $\alpha_{2}$ fixed) may be switched from synchronized to non-synchronized state if the network wiring changes ( $R$ changed) and vice verse.

The eigenvalue $\lambda_{l}$ is determined by the network structure (i.e., the coupling matrix $G$ ), and we mentioned in the above, the synchronization thresholds $\alpha_{1}$ and $\alpha_{2}$ depends on the properties of individual oscillators. Let us first consider unweighted network where the links have the same strength $w_{i j}=1$. For a regular network with only local connections, $\lambda_{2} \rightarrow 0$ so that the oscillators cannot be synchronized in large networks since the eigenratio $R \rightarrow \infty$ and the synchronization condition in Eq. (18) cannot be satisfied. Including a few shortcuts we can change the nonsynchronizable regular network to synchronizable smallworld networks because now $\lambda_{2}$ is non-zero and $R$ is finite. Randomizing connections of networks can improve synchronization because $\lambda_{2} \rightarrow k_{\min }$, the minimal degree of the networks. Therefore, increasing the average connectivity to have larger $k_{\text {min }}$ can also lead to enhanced synchronization in random networks. However, if the network possesses modular structure, adding links inside the modules will increase the modularity and enhance synchronization within the modules, but reduce the synchronizability of the whole network [55]. If the links are added to enhance the communication between the modules, synchronization of the whole network can be enhanced. In biological networks, a balance between these two situations seems to be crucial: the formation of network modules enables the modules to perform specialized functions through stronger synchronization within the modules. But some relatively weak degree of synchronization among the modules allows the system to perform integrated function from specialized subsystems.

The other limiting effect, the largest eigenvalue $\lambda_{N}$, is mainly determined by the maximal degree $k_{\max }$ of the network. Therefore it is difficult to synchronize degree heterogeneous networks such as the scale-free networks, if $\alpha_{2}$ is finite for given oscillators [56]. Nevertheless, if $\alpha_{2}$ is infinite, then heterogeneity degree will favor synchronization because the hubs will synchronize first [57].

The picture changes if we take the coupling weights into consideration. In a recent work, we have shown that for random enough networks without significant local connections and modular architectures, the eigenvalues are determined by the intensity of the nodes [58], in particular,

$\lambda_{2} \approx S_{\min }, \quad \lambda_{N} \approx S_{\max }$.

where $S_{\min }$ and $S_{\max }$ are the minimum and maximum of the intensity $S$ of the nodes respectively, defined as $S_{i}=\sum_{j} a_{i j} w_{i j}$, i.e., the total input weight of the a node from the other nodes in the network. Therefore, we can understand that the dynamical properties of the network can be changed by rearranging weights of the links. For example, if we normalize the weights of the nodes according to the degree $w_{i j}=1 / k_{i}$, the intensity becomes uniform $\left(S_{i}=1\right.$ for all the nodes) and the network can obtain the best synchronizability [59].

If the system cannot achieve the complete synchronization state because such a state is no longer a solution of the system in Eq. (16), for example, when the 
(a)

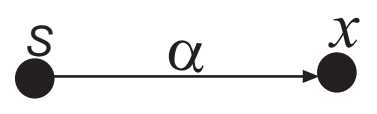

(b)

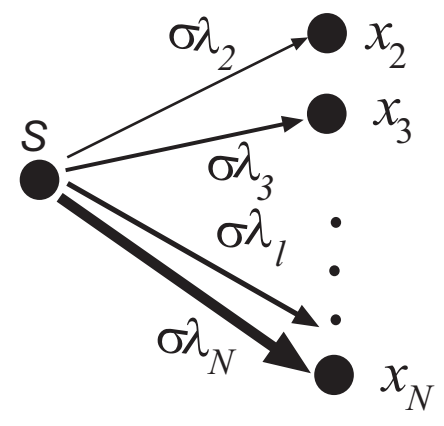

Fig. (7). Schematic plot of the eigenmode decomposition in network. (a) For 2 unidirectionally coupled nodes; (b) for a network of $N$ coupled nodes. From [53].

coupling is not strong enough, when the elements are nonidentical and diverse or when there are environmental perturbations, the dynamics of the network can display complicated patterns shaped by the underlying network structure [57]. We can obtain meaningful understanding by analyzing the eigenvectors of the coupling matrix. In scalefree network, the hubs having large degrees receive strong mean field influence from the rest of the networks thus can form effective synchronization clusters [57]. In modular networks, the dynamical interaction brings the nodes to form effective clusters of different levels depending on the strength of interactions [60].

Analyzing dynamical interaction in such large-scale networks from the viewpoint of synchronization can provide insights into various dynamical properties of large-scale biological networks. Recent investigation of synchronization dynamics of brain networks [61-63] have shed light on the challenging problem of the structure-function relationship in neural systems. The modelling and analysis using sophisticated neural population oscillators show a transition to synchronization very similar to that in Kuramoto model, even in the presence of strong noisy perturbations. The results show that noise-like dynamical fluctuations is structured which is consistent with experimental observation of complex functional networks in the brain [37-40]. Importantly, the synchronization analysis has shown that the functional connectivity is closely related to the underlying complex network organization, which would influence significantly the response of the brain neural systems to external stimulus. The interplay between structured background activity and the external signals should be relevant in many large-scale biological networks, such as metabolic networks, regulatory networks and protein interaction networks. For example, in metabolic networks, we typically consider the steady balance of the flux. Nevertheless, the flux may fluctuate in time, and the fluctuation of different metabolites is supposed to be correlated due to the underlying network interaction. Studying synchronization in networks can provide understanding of the relationship between such functional interaction patterns and the underlying network substrate, and provide insight into how biological system can function in a changing environment.

\section{CONCLUSION}

Timing in many biological systems is achieved by oscillation. Theoretical models, like Kuramoto model, can reveal some important features of synchronization of oscillators connected with simple or complex network architecture, which helps us achieve better understanding on oscillation and synchronization in biological systems.

Much effort is devoted and some interesting directions emerge recently. For synchronization that involves cell-cell communication, a small compound that can penetrate cell membranes is usually found. The concentration of such compound, i.e. the synchronizer, in the extracellular environment is closely related to the cell density. When cell density is high, the corresponding concentration is high and vice verse. Different extracellular concentrations sometimes can result in different behaviors of individual. In other words, this compound is the language used between individual cells. High concentration of synchronizer helps cells tell each other to have a population behavior. On the contrary, low concentration allows cells to tell each other to switch from population behavior into individual behavior. Such study [17, 64, 65], known as quorum sensing, may help us reveal the mystery of how bacteria sense cell density in order to choose between individual or population behavior. Furthermore, study on quorum sensing may even lead us to the understanding of the cell-cell communication in multicellular organisms. Besides quorum sensing, recent discussions indicate that the study on oscillation motif may help us to have a better global picture of the classified oscillators. Instead of studying a specified oscillator, oscillation motif, which is more general, focuses on the dynamics of a general class of oscillators. The oscillation pattern of one kind of such motifs, negative feedback loop, is well discussed lately, and simulation results of such model match experimental results from various different gene oscillators belonging to the same type of motif $[1,2]$. The dynamics of oscillation and synchronization of other genetic oscillators, classified into relaxation oscillator, smooth oscillator and stochastic oscillator, is also frequently discussed in recent years [66]. The oscillation motif study still mainly focuses on genetic oscillators. This type of study can help further discover the relationship between network topologies and dynamical behavior. Apart from the above directions, better quantitative understanding of the oscillatory behavior gives rise to the applications in synthetic 
biology. Synthetic genetic oscillators are rapidly produced in recent years $[64,67,68]$, which could make more practical usage in different fields.

All in all, study on oscillations and synchronization in biological systems has made great progress, both qualitatively and quantitatively, in the past few years. Many promising approaches are being applied and better understanding of this biological process may be around corner.

\section{ACKNOWLEDGEMENT}

We thank Prof. L.H. Tang for helpful and interesting discussion. The work is supported by Hong Kong Baptist University.

\section{REFERENCES}

[1] S. Pigolotti, S. Krishna and M.H. Jensen, "Oscillation patterns in negative feedback loops", Proc. Natl. Acad. Sci. USA, vol. 104, pp. 6533-6537, 2007.

[2] G. Tiana, S. Krishna, S. Pigolotti, M.H. Jensen and K. Sneppen, "Oscillations and temporal signalling in cells", Phys. Biol., vol. 4, no. 2, pp. R1-R17, 2007.

[3] R. May, "Stability and Complexity in Model Ecosystems", 2nd Ed., Princeton University Press, Princeton, 1975.

[4] M.A. Henson, D. Muller and M. Reuss, "Cell population modelling of yeast glycolytic oscillations", Biochem. J., vol. 368, Pt. 2, pp. 433-446, 2002.

[5] M.A. Henson, "Cell ensemble modeling of metabolic oscillations in continuous yeast cultures", Comput. Chem. Eng., vol. 29, no. 3, pp. 645-661, 2005.

[6] J. Wolf, and R. Heinrich, "Effect of cellular interaction on glycolytic oscillations in yeast: a theoretical investigation", Biochem. J., vol. 345, pp. 321-334, 2000.

[7] H.Y. Sohn and H. Kuriyama, "Ultradian metabolic oscillation of Saccharomyces cerevisiae during aerobic continuous culture: hydrogen sulphide, a population synchronizer, is produced by sulphite reductase”, Yeast, vol. 18, no. 2, pp. 125-135, 2001.

[8] H.Y. Sohn D.B. Murray and H. Kuriyama, "Ultradian oscillation of Saccharomyces cerevisiae during aerobic continuous culture: hydrogen sulphide mediates population synchrony", Yeast, vol. 16, no. 13, pp. 1185-1190, 2000.

[9] R.S. Lewis, "Calcium oscillations in T-cells: mechanisms and consequences for gene expression", Biochem. Soc. Trans., vol. 31, pp. 925-929, 2003.

[10] P. Mas, and M.J. Yanovsky, "Time for circadian rhythms: plants get synchronized”, Curr. Opin. Plant. Biol., vol. 12 , no.5, pp. 574579, 2009.

[11] C.H. Johnson, M. Egli, and P.L. Stewart, "Structural Insights into a Circadian Oscillator", Science, vol. 322, pp. 697-701, 2008.

[12] S.L. Harmer, "The circadian system in higher plants", Annu. Rev. Plant. Biol., vol. 60, pp. 357-77, 2009.

[13] T. Mori, D.R. Williams, M.O. Byrne, X. Qin, M. Egli, H.S. Mchaourab, P.L. Stewart and C.H. Johnson, "Elucidating the Ticking of an In vitro Circadian Clockwork", PLoS Biol., vol. 5, no.4, pp. e93, 2007.

[14] M. Nakajima, K. Imai, H. Ito, T. Nishiwaki, Y. Murayama, H. Iwasaki, T. Oyama and T. Kondo, "Reconstitution of Circadian Oscillation of Cyanobacterial KaiC Phosphorylation in vitro", Science, vol. 308, pp. 414-415, 2005.

[15] R. Kageyama, Y. Masamizu, and Y. Niwa, "Oscillator Mechanism of Notch Pathway in the Segmentation Clock", Devel. Dyn., vol. 236, no.6, pp. 1403-1409, 2007.

[16] Y. Bessho, and R. Kageyama, "Oscillations, clocks and segmentation", Curr. Opin. Genet. Dev., vol. 13, no.4, pp. 379-384, 2003.

[17] S. Dano, M.F. Madsen and P.G. Sorensen, "Quantitative characterization of cell synchronization in yeast", Proc. Natl. Acad. Sci. USA, vol. 104, pp. 12732-12736, 2007.

[18] M. Bier, B.M. Bakker and H.V. Westerhoff, "How Yeast Cells Synchronize their Glycolytic Oscillations: A Perturbation Analytic Treatment", Biophys. J., vol. 78, no.3, pp. 1087-1093, 2000.
[19] P. Richard, B.M. Bakker, B. Teusink, K. van Dam, and H.V. Westerhoff, "Acetaldehyde mediates the synchronization of sustained glycolytic oscillations in populations of yeast cells", Eur. J. Biochem., vol. 235, pp. 238-241, 1996.

[20] L. Kozma-Bognar, and K. Kaldi, "Synchronization of the fungal and the plant circadian clock by light", ChemBioChem, vol. 9, pp. 2565-2573, 2008.

[21] C. Huygens, The Pendulum Clock, Ames: Iowa State University Press, 1986.

[22] A.T. Winfree, "Biological rhythms and the behavior of populations of coupled oscillators", J. Theoret. Biol., vol. 16, no.1, pp. 15-42, 1967.

[23] A.T. Winfree, The Geometry of Biological Time, New York: Springer-Verlag Press, 1980

[24] Y. Kuramoto, Chemical oscillations, waves, and turbulence, New York: Springer-Verlag Press, 1984.

[25] J.A. Acebron, L.L. Bonilla, C.J.P. Vicente, F. Ritort and R. Spigler, "The Kuramoto model: A simple paradigm for synchronization phenomena", Rev. Mod. Phys., vol. 77, pp. 137-185, 2005.

[26] S.D. Monte, F. d'Ovidio, S. Dano and P.G. Sorensen, "Dynamical quorum sensing: Population density encoded in cellular dynamics", Proc. Natl. Acad. Sci. USA, vol. 104, pp. 18377-18381, 2007.

[27] R. Y. Moor, "Circadian Rhythms: Basic Neurobiology and Clinical Applications", Annu. Rev. Med., vol. 48, pp. 253-266, 1997.

[28] E.E. Abrahamson and R.Y. Moore, "Suprachiasmatic nucleus in the mouse: retinal innervation, intrinsic organization and efferent projections", Brain Res., vol. 916, pp. 172-191, 2001.

[29] S. Yamaguchi, H. Isejima, T. Matsuo, R. Okura, K. Yagita, M. Kobayashi and H. Okamura, "Synchronization of Cellular Clocks in the Suprachiasmatic Nucleus", Science, vol. 302, pp. 1408-1412, 2003.

[30] S.J. Kuhlman, R. Silver, J.L. Sauter, A. Bult-Ito and D.G. McMahon, "Phase Resetting Light Pulses Induce Per1 and Persistent Spike Activity in a Subpopulation of Biological Clock Neurons", J. Neurosci, vol. 23, pp. 1441-1450, 2003.

[31] J. Scannell, G.A.P.C. Burns, C.C. Hilgetag, M.A. O'Neil and M.P. Young, "The connectional organization of the cortico-thalamic system of the cat", Cereb. Cortex., vol. 9, pp. 277-299, 1999.

[32] O. Sporns, D.R. Chialvo, M. Kaiser and C.C. Hilgetag, "Organization, development and function of complex brain networks", Trends Cogn. Sci., vol. 8, pp. 418-425, 2004.

[33] O. Sporns and J.D. Zwi, "The small world of the cerebral cortex", Neuroinformatics, vol. 2, pp. 145-162, 2004.

[34] C.C. Hilgetag, G.A. Burns, M.A. O'Neill, J.W. Scannell, and M.P. Young, "Anatomical connectivity defines the organization of clusters of cortical areas in macaque monkey and cat", Philos. Trans. R. Soc. London, Ser. B., vol. 355, pp. 91-110, 2000.

[35] C. Hilgetag and M. Kaiser, "Clustered organization of cortical connectivity", Neuroinformatics, vol. 2, pp. 353-360, 2004.

[36] G. Buzsaki, Rhythms of the Brain. Oxford: Oxford University Press, 2006.

[37] C. Stam, "Functional connectivity patterns of human magnetoencephalographic recordings: A small-world network?" Neurosci. Lett., vol. 355, pp. 25-28, 2004.

[38] V.M. Eguiluz, D.R. Chialvo, G.A. Cecchi, M. Baliki and V.V. Apkarian, "Scale-free brain functional networks", Phys. Rev. Lett., vol. 94, 018102, 2005.

[39] R. Salvador, J. Suckling, M.R. Coleman, J.D. Pickard, D. Menon and E. Bullmore, "Neurophysiological architecture of functional magnetic resonance images of human brain", Cereb. Cortex., vol. 15, pp. 1332-1342, 2006.

[40] D.S. Bassett, A. Meyer-Lindenberg, S. Achard, T. Duke and E. Bullmore, "Adaptive reconfiguration of fractal small-world human brain functional networks", Proc. Natl. Acad. Sci. USA, vol. 103, pp. 19518-19523, 2006.

[41] D.S. Bassett and E. Bullmore, "Small-world Brain Networks", The Neuroscientist, vol. 12, pp. 521-523, 2006.

[42] E. Bullmore and O. Sporns, "Complex brain networks: graph theoretical analysis of the structural and functional systems", Nat. Rev. Neurosci., vol. 10, pp. 186-198, 2009.

[43] S.H. Strogatz, "Exploring complex networks", Nature (London), vol. 410, 268-276, 2001.

[44] R. Albert and A.-L. Barabasi, "Statistical mechanics of complex networks", Rev. Mod. Phys., vol. 74, pp. 47-97, 2002. 
[45] S. Boccaletti, V. Latora, Y. Moreno, M. Chavez and D.-U. Hwang, "Complex networks: Structure and dynamics", Phys. Rep., vol. 424, pp. 175-308, 2006.

[46] A.L. Barabasi and R. Albert, "Emergence of scaling in random networks", Science, vol. 286, pp. 509-512, 1999.

[47] D.J. Watts and S.H. Strogatz, "Collective dynamics of small-world networks", Nature (London), vol. 393, pp. 440-442, 1998.

[48] Y. Moreno and A.F. Pacheco, "Synchronization of Kuramoto oscillators in scale-free networks", Europhys. Lett., vol. 68, pp. 603-609, 2004.

[49] J.G. Restrepo, E. Ott and B.R. Hunt, "Onset of synchronization in large networks of coupled oscillators", Phys. Rev. E, vol. 71, pp. 036151, 2004.

[50] J. Gomez-Gardenes, Y. Moreno, A. Arenas, "Paths to synchronization on complex networks", Phys. Rev. Lett., vol. 98, pp. 034101, 2007.

[51] H. Kori and A. S. Mikhailov, "Entrainment of randomly coupled oscillator networks by a pacemaker", Phys. Rev. Lett., vol. 93, pp. 254101,2004

[52] H. Kori and A. S. Mikhailov, "Strong effects of network architecture in the entrainment of coupled oscillator systems", Phys. Rev. E, vol. 74, pp. 066115, 2006

[53] A. Arenas, A. Diaz-Guilera, J. Kurths, Y. Morenob and C.S. Zhou, "Synchronization in complex networks", Phys. Rep., vol. 469, pp. 93-153, 2008.

[54] M. Barahona and L.M. Pecora, "Synchronization in small-world systems", Phys. Rev. Lett., vol. 89, pp. 054101, 2002.

[55] L. Huang, K. Park, Y.-C. Lai, L. Yang and K. Yang, "Abnormal synchronization in complex clustered networks", Phys. Rev. Lett., vol. 97, pp. 164101, 2006.

[56] T. Nishikawa, A.E. Motter, Y.-C. Lai and F.C. Hoppensteadt, "Heterogeneity in oscillator networks: Are smaller worlds easier to synchronize?" Phys. Rev. Lett., vol. 91, pp. 014101, 2003.

[57] C.S. Zhou and J. Kurths, "Hierarchical synchronization in complex networks with heterogeneous degrees", Chaos, vol. 16, pp. 015104, 2006.
[58] C.S. Zhou, A.E. Motter and J. Kurths, "Universality in the synchronization of weighted random networks", Phys. Rev. Lett., vol. 96, pp. 034101, 2006

[59] A.E. Motter, C.S. Zhou and J. Kurths, "Enhancing complexnetwork synchronization", Europhys. Lett., vol. 69, pp. 334-340, 2005.

[60] A. Arenas, A. Diaz-Guilera and C.J. Perez-Vicente, "Synchronization reveals topological scales in complex networks", Phys. Rev. Lett., vol. 96, pp. 114102, 2006.

[61] C.S. Zhou, L. Zemanova, G. Zamora, C. C. Hilgetag and J. Kurths, "Hierarchical organization unveiled by functional connectivity in complex brain networks", Phys. Rev. Lett., vol. 97, pp. 238103 , 2006.

[62] L. Zemanova, C. Zhou and J. Kurths, "Structural and functional clusters of complex brain networks", Phys. D, vol. 224, pp. 202212, 2006.

[63] C. Zhou, L. Zemanova, G. Zamora, C.C. Hilgetag and J. Kurths, "Structure function relationship in complex brain networks expressed by hierarchical synchronization", New J. Phys., vol. 9, pp. 178, 2007.

[64] D. McMillen, N. Kopell, J. Hasty, and J.J. Collins, "Synchronizing genetic relaxation oscillators by intercell signaling", Proc. Natl. Acad. Sci. USA, vol. 99, pp. 679-684, 2002.

[65] S. Hooshangi, and W.E. Bentley, "From unicellular properties to multicellular behavior: bacteria quorum sensing circuitry an applications", Curr. Opin. Biotechnol., vol. 19, pp. 550-555, 2008.

[66] T.S. Zhou, J.J. Zhang, Z.J. Yuan and L.N. Chen, "Synchronization of genetic oscillators", Chaos, vol. 18, no. 3, pp. 037126, 2008.

[67] D. Gonze, "Coupling oscillations and switches in genetic networks", Biosystems, vol. 99, no.1, pp. 60-69, 2010

[68] M.B. Elowitz and S. Leibler, "A synthetic oscillatory network of transcriptional regulators", Nature, vol. 403, pp. 335-338, 2000.

(c) Li and Zhou; Licensee Bentham Open.

This is an open access article licensed under the terms of the Creative Commons Attribution Non-Commercial License (http://creativecommons.org/licenses/by-nc/3.0/) which permits unrestricted, non-commercial use, distribution and reproduction in any medium, provided the work is properly cited. 